\title{
ELECTRON DISPERSION IN LIQUID ALKALI METALS
}

\author{
P.N. GajJar, B.Y. Thakore and A.R. Jani \\ Department of Physics, Sardar Patel University \\ Vallabh Vidyanagar 388 120, Gujarat, India
}

(Received April 27, 1998)

\begin{abstract}
A local pseudopotential in the second-order perturbation theory is used to study the electron dispersion relation, the Fermi energy and deviation in the Fermi energy from free electron value for the liquid alkali metals. The influence of the five different forms of the local field correction functions on the aforesaid electronic properties is examined explicitly, which reflects the varying effects of screening. The depth of the first negative hump in the electron dispersion curves of the liquid alkali metals increases in the order $\mathrm{Na}, \mathrm{K}, \mathrm{Rb}, \mathrm{Cs}$.
\end{abstract}

PACS numbers: $61.20 .-\mathrm{p}, 61.25 . \mathrm{Mv}, 63.20 . \mathrm{Dj}$

\section{Introduction}

The pseudopotential based investigation of the Fermi surface and its distortion from free-electron value for the metals in the solid phase are quite often and well recognized $[1,2]$. However, the attempts of studying the Fermi energy and its deviation from the free electron value for liquid metals are very rare and not exhaustive $[3,4]$. For liquid metals, it was observed that there exist deviations in the electron dispersion from the free electron value. It was also found that the maximum deviation takes place in the vicinity of the first spherical Brillouin zone. This region lies nearly at half of the distance of the first peak in the structure factor. Srivastava [3] has reported the electron dispersion and the Fermi energy of some simple liquid metals. He has also reported the deviation in the Fermi energy from the free electron value at the Fermi level for the liquid alkali metals [4]. Present article deals with the computation of the electron dispersion, Fermi energy and deviation in the Fermi energy of liquid alkali metals with the aim to explore the use of our well established empirical form of the local pseudopotential [1, 2, 5-7] for understanding the behavior of these properties. The local pseudopotential used to explain the electron-ion interaction in liquid metals is of the form (in Rydberg units) $[1,2,5-7]$ :

$$
W_{\mathrm{B}}(q)=-\frac{8 \pi Z}{\Omega_{0} q^{2}}\left(\cos q r_{\mathrm{c}}-\frac{q^{2} r_{\mathrm{c}}^{2}}{1+q^{2} r_{\mathrm{c}}^{2}}\right) .
$$


Here $Z$ is the valency, $\Omega_{0}$ - the atomic volume, $q-$ the wave vector and $r_{\mathrm{c}}-$ the parameter of the model potential. Since the parameter of the potential determines the size of the effective core region and as soon as the size will change, the interaction and hence the pseudopotential will change. Thus the determination of the parameter bears prime importance. Instead of fitting the parameter of the model potential with any observed physical property, the well recognized method $[1,2,5-7]$ of $q=q_{0}$ value is used to determine the parameter of the potential. The requirement to determine the parameter $r_{\mathrm{c}}$ from $q_{0}$ value leads to the condition $q_{0} r_{\mathrm{c}}=1.0301[1,2,5-7]$.

The choice of pseudopotential form factor is certainly an important consideration in the study of metallic properties and its actual form is much more sensitive to the choice of the dielectric function of the electron gas. Hence, the purpose of this paper is not only to generate the electron dispersion curves, Fermi energy, and deviation in the Fermi energy from its free electronic value, but also to see the influence of various local-field correction functions in the screening. For this, we incorporated five different forms of the exchange and correlation functions, viz. Hartree (H) [8], Hubbard-Sham (HS) [9], Vashishta-Singwi (VS) [10], Taylor (T) [11] and Ichimaru-Utsumi (IU) [12], with special attention to see the effect of IU [12] function, since this function is not exhaustively used in the pseudopotential calculations so far. IU [12] screening function reproduces accurately the Monte Carlo results as well as those of the microscopic calculations. It also satisfies self-consistency conditions in the compressibility sum rule and short-range correlations. A notable feature in this function is its involvement of the logarithmic singularity at $q=2 k_{\mathrm{F}}$ and the accompanying peak at $q \approx 1.94 k_{\mathrm{F}}$.

\section{Electronic structure}

In the second-order perturbation theory, involving pseudopotential, the electronic structure of a liquid metal is given as $[3,4]$ :

$$
E(k)=E_{0}(k)+E_{1}(k)+E_{2}(k),
$$

where

$$
\begin{aligned}
& E_{0}(k)=\hbar^{2} k^{2} / 2 m, \\
& E_{1}(k)=N\langle k|w(q)| k\rangle,
\end{aligned}
$$

and

$$
E_{2}(k)=\frac{2 m}{\hbar^{2}} \sum_{q} \frac{S(q) S^{*}(q)|w(q)|^{2}}{k^{2}-|k+q|^{2}} .
$$

Using the liquid structure factor $a(q)=N|S(q)|^{2}$, we rewrite Eq. (2) in the following form $[3,4]$ :

$$
E(k)=\frac{\hbar^{2} k^{2}}{2 m}+\frac{2 m}{\hbar^{2}}\left[\sum_{q} \frac{a(q)|w(q)|^{2}}{k^{2}-|k+q|^{2}}-\frac{a(q)|w(q)|^{2}}{q^{2}}\right] .
$$

For liquid analog this equation is restructured as [3]:

$$
E(k)=\hbar^{2} k^{2} / 2 m+\Delta(k)-\Delta(0)
$$


with

and

$$
\Delta(k)=\frac{3 Z}{8 k_{\mathrm{F}} E_{\mathrm{F}} k} \int_{0}^{\infty} a(q) w^{2}(q) q \ln \left|\frac{2 k+q}{2 k-q}\right| \mathrm{d} q,
$$

$$
\Delta(0)=\frac{3 Z}{2 k_{\mathrm{F}} E_{\mathrm{F}}} \int_{0}^{\infty} a(q) w^{2}(q) \mathrm{d} q .
$$

Here $k_{F}, E_{\mathrm{F}}, a(q)$ and $w(q)$ are the Fermi momentum, Fermi energy, static structure factor, and pseudopotential form factor, respectively. From Eq. (7), the electron dispersion $\Delta E(k)$ for the liquid metals is given as

$$
\Delta E(k)=\Delta\left(k_{\mathrm{F}}\right)-\Delta(0) \text {. }
$$

At the Fermi level, $k=k_{\mathrm{F}}$, Eqs. (7) to (10) take the value [3, 4]:

$$
E_{\mathrm{F}}\left(k_{\mathrm{F}}\right)=\hbar^{2} k_{\mathrm{F}}^{2} / 2 m+\Delta\left(k_{\mathrm{F}}\right)-\Delta(0),
$$

with

$$
\Delta\left(k_{\mathrm{F}}\right) \doteq \frac{3 Z}{8 k_{\mathrm{F}}^{2} E_{\mathrm{F}}} \int_{0}^{2 k_{\mathrm{F}}} a(q) w^{2}(q) q \ln \left|\frac{2 k_{\mathrm{F}}+q}{2 k_{\mathrm{F}}-q}\right| \mathrm{d} q,
$$

and

$$
\Delta(0)=\frac{3 Z}{2 k_{\mathrm{F}} E_{\mathrm{F}}} \int_{0}^{2 k_{\mathrm{F}}} a(q) w^{2}(q) \mathrm{d} q .
$$

Hence the cieviation in the Fermi energy $\Delta E_{\mathrm{F}}$ from free electron value at the Fermi level is given by

$$
\Delta E_{\mathrm{F}}=\Delta\left(k_{\mathrm{F}}\right)-\Delta(0) \text {. }
$$

\section{Results and discussion}

From the set of Eqs. (7) to (10), we computed the electron dispersion in liquid alkali metals which are shown in Figs. 1-5. Theoretical structure factor evaluated by using Percus-Yevick hard-core (hard-sphere) approximation [13] was used in the calculations. Figures 1-5 reflect that the inclusion of various local-field corrections influence strongly the outcome. Except for $\mathrm{Na}$, the first negative maxima in the electron dispersion curves due to HS [9], VS [10], and IU [12] screening function fall between those of due to $\mathrm{H}[8]$ and $\mathrm{T}[11]$ screening function. While in $\mathrm{Na}$, the first negative maxima due to $H[8]$, HS [9], and VS [10] local field correction are lying between those obtained by IU [12] and T [11] local field correction functions. It is found that liquid Cs has the largest negative hump in the electron dispersion among all alkalis. The depth of the negative hump in the electron dispersion of liquid alkali metals increases in the order $\mathrm{Na}, \mathrm{K}, \mathrm{Rb}$, and Cs. This is a clear demonstration of the fact that the strength of the potential increases as we move from sodium to cesium. However, the $\mathrm{Li}$ shows larger negative hump than $\mathrm{Na}, \mathrm{K}$, and $\mathrm{Rb}$. This could be due to non-orthogonalization of $p$-component of the conduction electrons. The oscillatory behavior in the $\Delta E(k) \rightarrow k$ curves indicates that the electron dispersion may have an important effect on the electronic properties of liquid metals. 


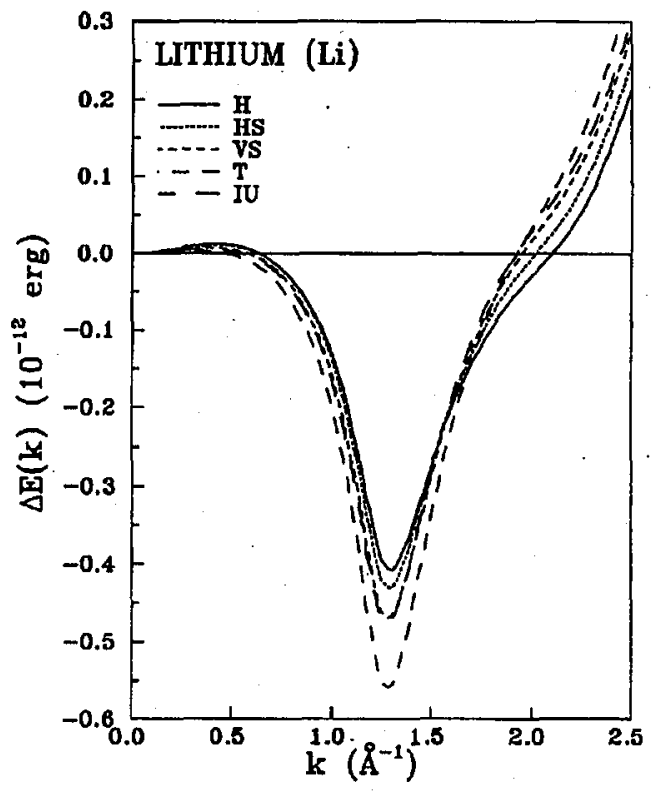

Fig. 1. Electron dispersion curves for liquid Li.

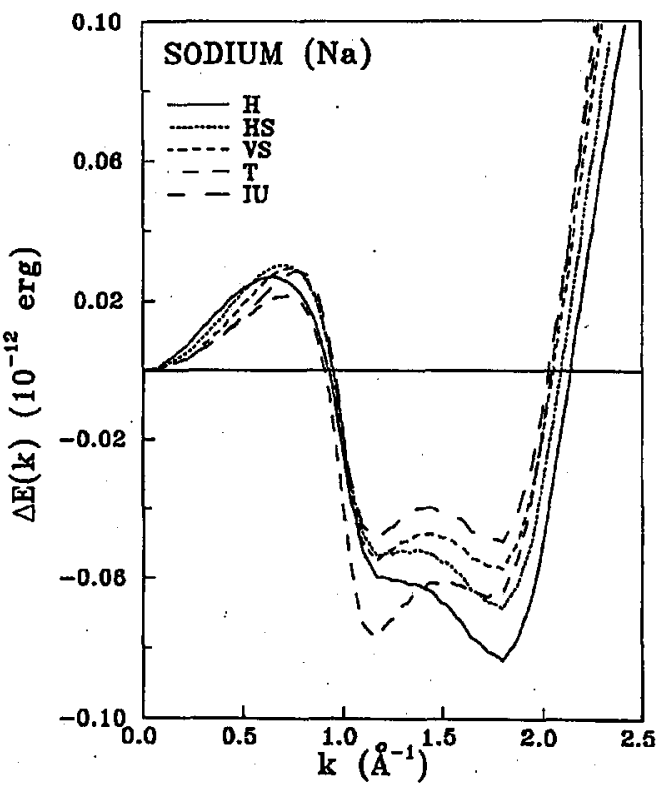

Fig. 2. Electron dispersion curves for liquid Na. 


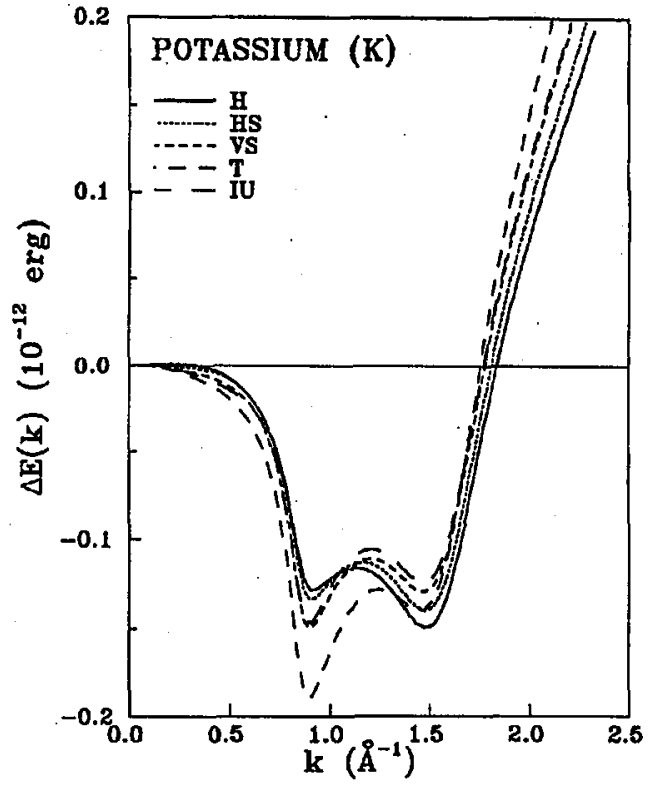

Fig. 3. Electron dispersion curves.for liquid K.

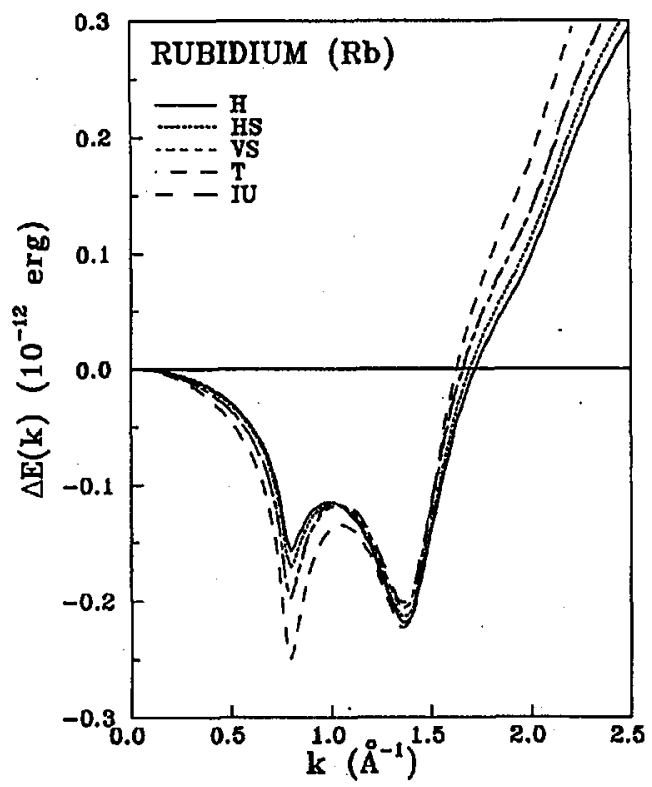

Fig. 4. Electron dispersion curves for liquid Rb. 


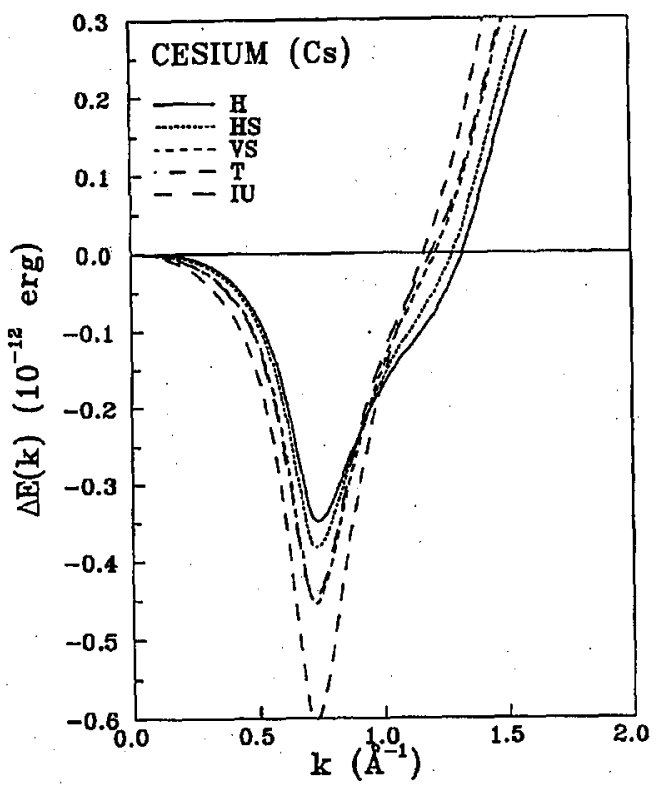

Fig. 5. Electron dispersion curves for liquid Cs.

TABLE

Fermi energy $\left(-E_{\mathrm{F}}\right.$ in $\left.10^{-12} \mathrm{erg}\right)$ for liquid alkali metals.

\begin{tabular}{|c|c|c|c|c|c|c|c|c|c|}
\hline \multirow[t]{2}{*}{ Metal } & \multicolumn{5}{|c|}{ Present work with different screening } & \multicolumn{4}{|c|}{ Other results [3] } \\
\hline & $\mathrm{H}[8]$ & HS [9] & VS [10] & $\mathrm{T}[11]$ & IU [12] & & & & \\
\hline \multirow[t]{2}{*}{$\mathrm{Li}$} & 7.1149 & 7.0961 & 7.0805 & 7.0662 & 7.0633 & 7.2965 & 6.9623 & 7.0861 & 7.4199 \\
\hline & & & & & & $\dot{7} .2530$ & 6.8584 & 7.0021 & 7.3989 \\
\hline \multirow[t]{2}{*}{$\mathrm{Na}$} & 4.7388 & 4.7199 & 4.7057 & 4.7008 & 4.6934 & 5.0477 & 4.9283 & 4.9578 & 5.0879 \\
\hline & & & & & & 5.0566 & 4.9170 & 4.9544 & 5.0986 \\
\hline \multirow[t]{2}{*}{$\mathrm{K}$} & 3.3493 & 3.3404 & 3.3328 & 3.3306 & 3.3270 & 3.3030 & 3.2184 & 3.2081 & 3.3105 \\
\hline & & & & & & 3.2811 & 3.1559 & 3.1701 & 3.2922 \\
\hline \multirow[t]{2}{*}{$\mathrm{Rb}$} & 2.7919 & 2.7802 & 2.7699 & 2.7668 & 2.7620 & 2.8390 & 2.7448 & 2.7697 & 2.8755 \\
\hline & & & & & & 2.8662 & 2.7703 & 2.7755 & 2.9007 \\
\hline \multirow[t]{2}{*}{ Cs } & 2.2119 & 2.1977 & 2.1783 & 2.1592 & 2.1582 & 2.4294 & 2.3488 & 2.3293 & 2.4617 \\
\hline & & & & & & 2.4396 & 2.3559 & 2.3354 & 2.4724 \\
\hline
\end{tabular}


Equations (11) to (14) are employed to calculate the Fermi energy and the deviation in the Fermi energy from the free electron value at the Fermi level. The calculated values of the Fermi energy $E_{\mathrm{F}}$ for the liquid alkali metals are listed in Table along with other such findings [3] which fully supports the present values for all the alkali metals. It is to be noted that different forms of the local-field correction functions [8-12] to incorporate the exchange and correlation effects in the screening reduces the value of Fermi energy in comparison with that obtained by static Hartree dielectric function [8] (which does not include any exchange or correlation effects). Figure 6 shows the trend of the evaluated change in the Fermi energy $E_{\mathrm{F}}$ from free-electron value versus atomic volume $\Omega_{0}$ for the liquid alkalis. The effect of the various local-field correction function is distinct and more transparent on $\Delta E_{\mathrm{F}}$. Among the alkali metals, minimum deviation in the Fermi energy at the Fermi level is obtained for. liquid potassium and the maximum deviation in the Fermi energy at the Fermi level is obtained for liquid lithium.

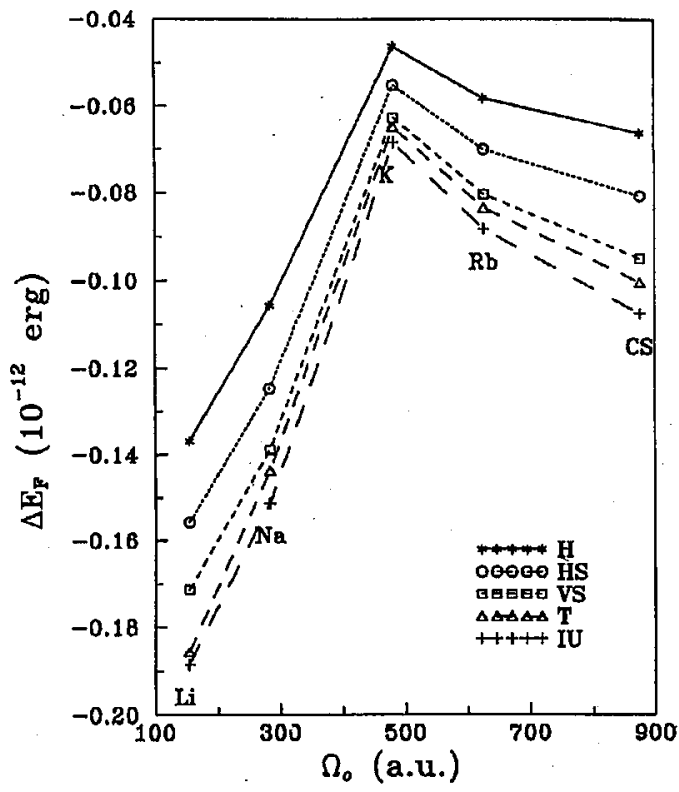

Fig. 6. Deviation in the Fermi energy versus atomic volume.

From the present investigation we found that IU [12] local field correction function produces satisfactory results and behaves equally good in comparison to other screening functions [8-11] employed in the present work. We conclude with stating that in the pseudopotential based calculations one has to employ the IU [12] screening function to test the validity and usefulness of the model potential. Finally, we note that similar work could be done for other polyvalent liquid metals to arrive at confirm conclusion. Such work is under progress and reported in due course of time. 


\section{References}

[1] P.N. Gajjar, B.Y. Thakore, A.R. Jani, Solid State Commun. 100, 785 (1996).

[2] P.N. Gajjar, B.Y. Thakore, J.S. Luhar, A.R. Jani, Indian J. Pure Appl. Phys. 33, 309 (1995).

[3] S.K. Srivastava, J. Phys. Chem. Solids 36, 993 (1975).

[4] S.K. Srivastava, Ram Dawar, Indian J. Pure Appl. Phys. 31, 520 (1993).

[5] P.N. Gajjar, B.Y. Thakore, J.S. Luhar, A.R. Jani, Acta Phys. Pol. A 86, 369 (1994).

[6] R.S. Avte, P.N. Gajjar, A.R. Jani, Phys. Status Solidi B 176, K59 (1993).

[7] A.R. Jani, P.N. Gajjar, H.K. Patel, Phys. Status Solidi B 169, K105 (1992).

[8] W. Harrison, Pseudopotentials in the Theory of Metals, W.A. Benjamin, New York 1967.

[9] J. Hubbard, Proc. R. Soc. A 234, 336 (1958); L.J. Sham, Proc. R. Soc. A 283, 33 (1965).

[10] P. Vashishta, K.S. Singwi, Phys. Rev. B 6, 875 (1972).

[11] R. Taylor, J. Phys. F 8, 1699 (1978).

[12] S. Ichimaru, K. Utsumi, Phys. Rev. B 24, 3220 (1981).

[13] J.K. Percus, G.J. Yevick, Phys. Rev. 110, 1 (1958). 